\title{
Clinical assessment of three-dimensional printing assisted osteotomy guide plates in precise osteotomy of adult talipes equinovarus
}

\section{Yuan-Wei Zhang}

Jiangxi Provincial People's Hospital Affiliated to Nanchang University

\section{Liang-Yu Xiong}

Zhangshu Municipal People's Hospital

\section{Zu-Tai Huang}

Jiangxi Provincial People's Hospital Affiliated to Nanchang University

\section{Wen-Cheng Gao}

Dongguan Houjie Town People's Hospital

\section{Xin Xiao}

Medical college of Nanchang University

\section{Su-Li Zhang}

Wujin Hosptial Affiliated to Jiangsu University

\section{Wen-Yan Ni}

Wujin Hospital Affiliated to Jiangsu University

\section{Liang Deng ( $\nabla$ dengliang001137@163.com )}

Jiangxi Provincial People's Hospital Affiliated to Nanchang University https://orcid.org/0000-00027221-9146

\section{Research article}

Keywords: three-dimensional printing, adult talipes equinovarus, triple arthrodesis, osteotomy guide plate

Posted Date: May 18th, 2020

DOI: https://doi.org/10.21203/rs.2.24195/v2

License: (c) (i) This work is licensed under a Creative Commons Attribution 4.0 International License.

Read Full License 
The authors have withdrawn this preprint from Research Square 